Article

\title{
Cytotoxic Effects of Newly Synthesized Heterocyclic Candidates Containing Nicotinonitrile and Pyrazole Moieties on Hepatocellular and Cervical Carcinomas
}

\author{
Amira A. El-Sayed ${ }^{1, *}$, Abd El-Galil E. Amr ${ }^{2,3, * \mathbb{C}}$, Ahmed K. EL-Ziaty ${ }^{1}$ and \\ Elsayed A. Elsayed ${ }^{4,5}$ \\ 1 Laboratory of Synthetic Organic Chemistry, Chemistry Department, Faculty of Science, Ain Shams \\ University, Abbassia, Cairo 11566, Egypt; ahm512@gmail.com \\ 2 Pharmaceutical Chemistry Department, Drug Exploration \& Development Chair (DEDC), College of \\ Pharmacy, King Saud University, Riyadh 11451, Saudi Arabi \\ 3 Applied Organic Chemistry Department, National Research Center, Cairo, Dokki 12622, Egypt \\ 4 Zoology Department, Bioproducts Research Chair, Faculty of Science, King Saud University, Riyadh 11451, \\ Saudi Arabiap; eaelsayed@ksu.edu.sa \\ 5 Chemistry of Natural and Microbial Products Department, National Research Centre, Dokki 12622, \\ Cairo, Egypt \\ * Correspondence: aamr@ksu.edu.sa (A.E.-G.E.A.); amira_aa47@hotmail.com (A.A.E.-S.); \\ Tel.: +966-543074312 (A.E.-G.E.A.); +201-006532767 (A.A.E.-S.)
}

Academic Editor: Qiao-Hong Chen

Received: 28 April 2019; Accepted: 16 May 2019; Published: 22 May 2019

\begin{abstract}
In this study, a series of newly synthesized substituted pyridine 9, 11-18, naphthpyridine derivative 10 and substituted pyrazolopyridines $19-23$ by using cycnopyridone 8 as a starting material. Some of the synthesized candidates are evaluated as anticancer agents against different cancer cell lines. In vitro cytotoxic activities against hepatocellular and cervical carcinoma cell lines were evaluated using standard MTT assay. Different synthesized compounds exhibited potential in vitro cytotoxic activities against both HepG2 and HeLa cell lines. Furthermore, compared to standard positive control drugs, compounds 13 and 19 showed the most potent cytotoxic effect with $\mathrm{IC}_{50}$ values of $8.78 \pm 0.7,5.16 \pm 0.4 \mu \mathrm{g} / \mathrm{mL}$, and $15.32 \pm 1.2$ and $4.26 \pm 0.3 \mu \mathrm{g} / \mathrm{mL}$ for HepG2 and HeLa cells, respectively.
\end{abstract}

Keywords: cyanopyridone; substituted pyridine; pyridotriazine; pyrazolopyridine; thioxotriazopyridine; anticancer activity; HepG2; HeLa

\section{Introduction}

Multicomponent reactions (MCR) "in which three or more starting materials react to form a product" play a significant role in the synthesis of heterocyclic compounds with pharmaceutical and chemical importance [1]. Several nicotinonitriles have been constructed via (MCR) and showed antitumor [2], antimicrobial [3], and antioxidant [4] activities. Also nicotinonitriles have been utilized as a scaffold for the synthesis of heterocyclic compounds containing a pyridine moiety with antimicrobial and antiviral activities [5]. A series of nicotinonitriles 1-3 (Figure 1) and have been synthesized and anti-proliferative [6], anti-Alzheimer's [7], and anti-inflammatory [8] activities. 


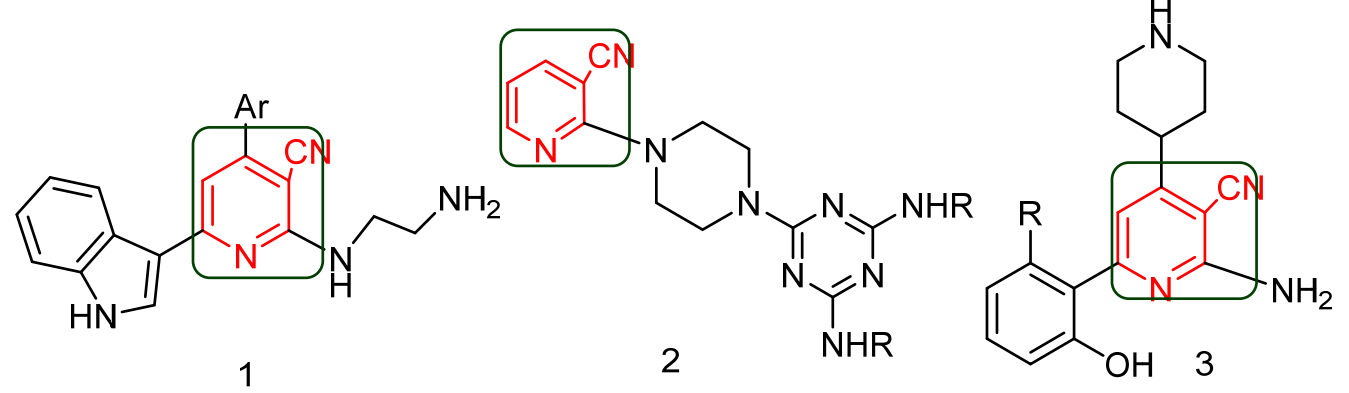

Figure 1. Nicotinonitriles with anti-proliferative, anti-Alzheimer's anti-inflammatory activities.

The pyrazole moiety is both pharmacologically and medicinally significant [9]. A series of pyrazoles 4-7 (Figure 2) has been reported as anti-inflammatory activity by Bekhit et al. [10], they observed that the synthesized pyrazoles showed more anti-inflammatory activity than the standard indomethacin [11]. Trisubstituted pyrazoles have been constructed by Christodoulou et al. (2010) [11] and evaluated as anti-angiogenic agents; these derivatives showed a potent anti-angiogenic efficacy and moreover inhibited the growth of Mammary gland breast cancer (MCF-7) and cervical carcinoma (Hela) [12]. Recently novel derivatives of pyrazoles $\mathbf{5 , 6}$ have been prepared as antimicrobial [13] and anticonvulsant [14] agents. The pyrazole 7 has been prepared by Bonesi et al. (2010) [15] and showed effective Angiotensin -1-Converting Enzyme (ACE) inhibitor activity [15].

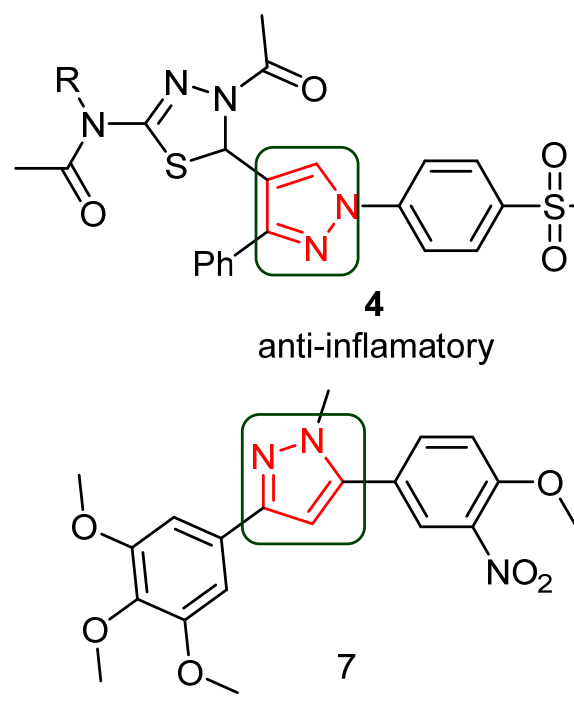

(ACE) inhibator<smiles>Oc1ccc(NC2CC3CCCC(C2)N3Cc2ccc(Cl)cc2)cc1</smiles>

Antimicrobial<smiles>COc1ccccc1C1(C(C)=O)CC2CCC(C1)N2C(C)=O</smiles>

Anticonvulsant

Figure 2. Pyrazoles as anti-inflammatory antimicrobial and anticonvulsant activities.

Based on the previous facts about the importance of pyrazoles and nicotinonitriles in medicinal chemistry, we have herein synthesized of some novel heterocyclic candidates containing nicotinonitrile and pyrazole moieties and tested their anticancer activity.

\section{Results}

\subsection{Chemistry}

The nicotinonitriles were obtained by two different ways, from the reaction of chalcone with ethylcyanoacetate, ammonium acetate and drops of piperidine as a base and from one pot four components reaction of methylketone, aldehyde, ethylcyanoacetate, ammonium acetate and drops of piperidine as a base [15]. In prolongation of our work in the synthesis of heterocyclic compounds and evaluation of their medicinal importance [16-27] and based on the literature survey about the 
pharmacological and medicinal importance of pyrazoles and nicotinonitriles, we have devoted our efforts to design and synthesize novel heterocyclic compounds containing pyrazol and nicotine-nitrile moieties, 4-(3-(4-fluorophenyl)-1-phenyl-1H-pyrazol-4-yl)-2-hydroxy-6-(naphthalen-1-yl)-nicotinenitrile 8 has been obtained by reacting of 1-acetylnaphthalene (A), 3-(4-fluorophenyl)-1-phenyl-1Hpyrazole-4-carbaldehyde (B), ethyl 2-cyanoacetate, ammonium acetate and piperidine (Scheme 1).<smiles>CCOC(=O)CC(N)=O</smiles>

(B)

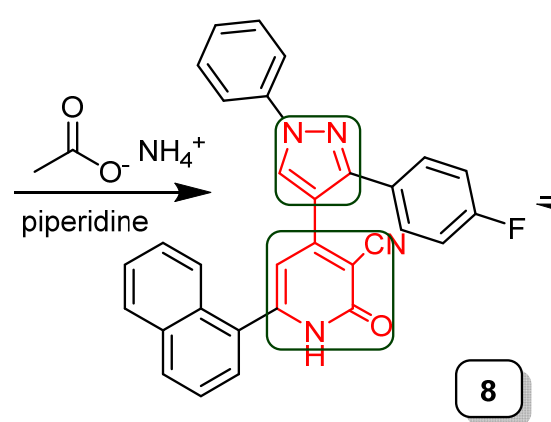

8

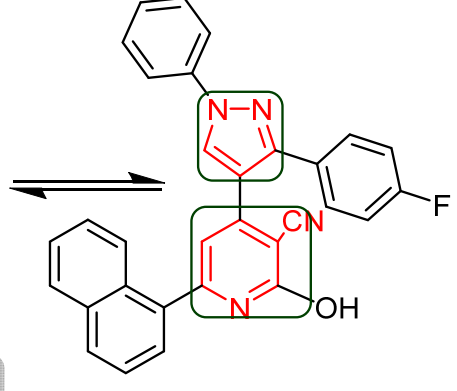

Scheme 1. Synthesis of compound $\mathbf{8}$ as starting material.

The structure of the nicotinonitrile 8 has been confirmed from its spectral data. IR spectrum showing absorption frequencies at $v 3159 \mathrm{~cm}^{-1}, 2220 \mathrm{~cm}^{-1}$ and $v 1647 \mathrm{~cm}^{-1}$ for $\mathrm{OH}, \mathrm{C} \equiv \mathrm{N}$ and $\mathrm{C}=\mathrm{N}$ groups, respectively. Also, ${ }^{1} \mathrm{H}-\mathrm{NMR}$ spectrum of the assigned compound displayed signals at $\delta 12.89 \mathrm{ppm}$ (disappeared with $\mathrm{D}_{2} \mathrm{O}$ ) corresponding to acidic $\mathrm{OH}$. A compelling evidence for the structure of 8 was provided by ${ }^{13} \mathrm{C}-\mathrm{NMR}$ spectrum that showed a singlet signal at $\delta 149.8,139.3$ and $139.3 \mathrm{ppm}$ for $\mathrm{C}-\mathrm{OH}, \mathrm{C}=\mathrm{N}$ and $\mathrm{C} \equiv \mathrm{N}$ groups respectively. Mass spectra of $\mathbf{8}$ showed $\left[\mathrm{M}^{+}\right]$at $m / z(\%)$ 482 (22). Treatment of 8 with ethylchloroacetate afforded compound $\mathbf{9}$, which was hydrazinolysis with $\mathrm{NH}_{2} \mathrm{NH}_{2}$ to give the corresponding cyclized product 10 .

Remediation of the nicotinonitrile derivative 8 with malononitrile in the presence of few drops of piperidine afforded 1,8-naphthyridine-3-carbonitrile derivative 11. Chlorination of 8 by a mixture of $\left(\mathrm{POCl}_{3} / \mathrm{PCl}_{5}\right)$ afforded 2-chloronicotinonitrile derivative 12, which was reacted with malono nitrile as a carbon nucleophile gave the nicotinonitrile derivative $\mathbf{1 3}$. Reaction of $\mathbf{1 2}$ with primary and secondary amines, namely, $o$-aminothiophenol, morpholine, 1-methylpiperazine and hydrazine hydrate gave novel nicotinonitriles 14, 15a, b and $\mathbf{1 6}$ (Scheme 2). The mechanism formation route of compound 11 has been shown in Figure 3.

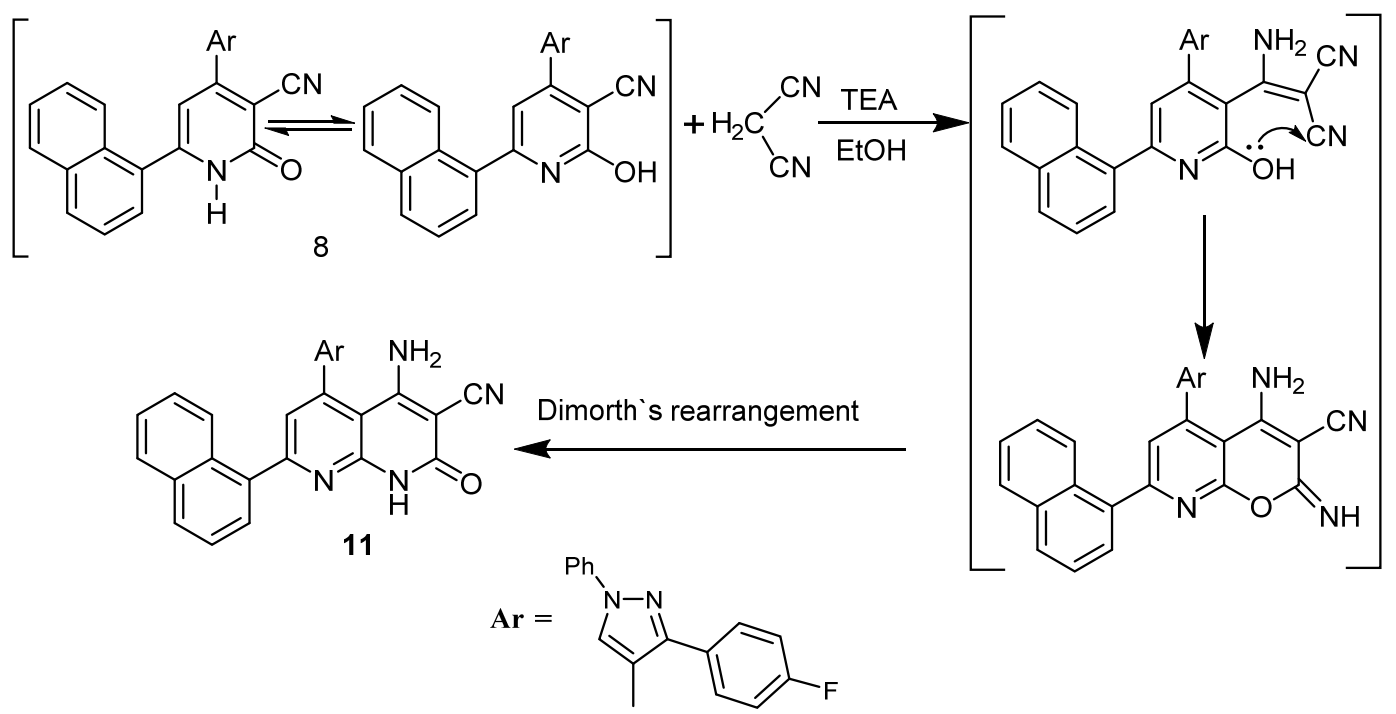

Figure 3. The mechanism formation route of compound $\mathbf{1 1}$. 


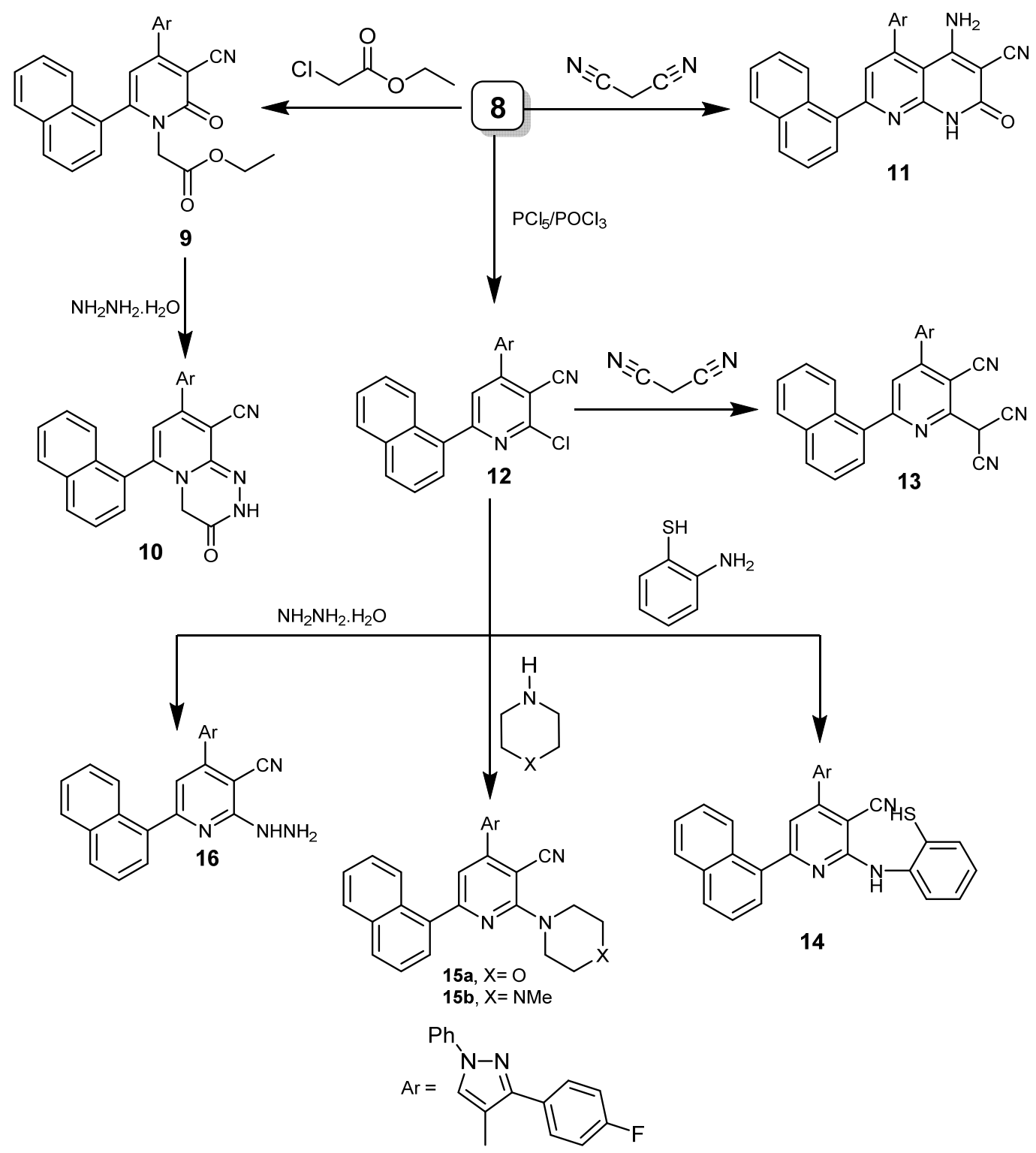

Scheme 2. Synthetic route for compounds 9-16.

Compound 16 was utilized as a building block for novel nicotinonitriles containing two pyrazole moieties. 2-Pyrazolyl nicotinonitrile derivatives 17 and 18 were prepared by treatment of 16 with acetyl acetone and 4,4,4,-trifluoro-1-(thiophen-2-yl)butane-1,3-dione, respectively. Treatment of $\mathbf{1 6}$ with acetic anhydride and acetic acid afforded pyrazolopyridine derivative 19. The derivative 16 was treated with acetic anhydride to afford the $N$-acetyl pyrazolopyridine as a sole product $\mathbf{2 0}$. The structure of compound 20 was confirmed chemically by acetylation of the amino pyrazopyridine $\mathbf{1 9}$ (Scheme 3).

Treatment of $\mathbf{1 6}$ with 4-chlorobenzaldehyde and/or tetrachlorophthalic anhydride in the presence of acetic acid afforded the cyclized 19 followed by condensation to give the Schiff's base $\mathbf{2 1}$ and tetra chloroisoindoline 22, respectively. The structures of $\mathbf{2 1}$ and 22 were confirmed chemically by condensation of compound 19 with 4-chlorobenzaldehyde and/or tetrachlorophthalic anhydride to provide compounds $\mathbf{2 1}$ and 22, respectively. Treatment of hydrazinyl derivative $\mathbf{1 6}$ with $\mathrm{CS}_{2}$ in the presence of alcoholic $\mathrm{KOH}$ provided thioxotriazolo pyridine derivative $\mathbf{2 3}$ (Scheme 3). 


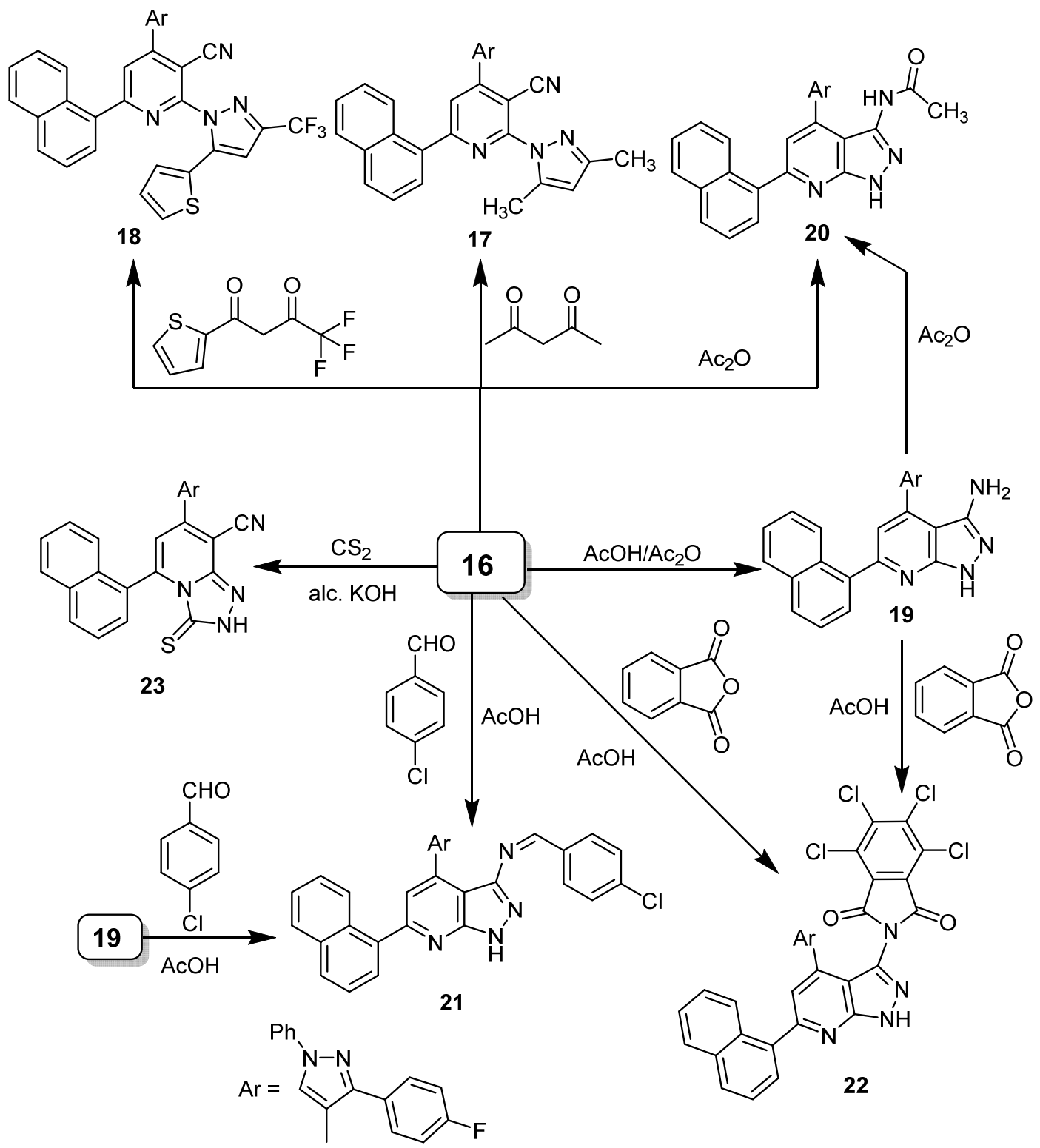

Scheme 3. Synthetic route for compounds 17-23.

\subsection{Cytotoxic Activity}

The newly synthesized compounds were screened for their anticancer potentials against hepatocellular carcinoma HepG2 and cervical carcinoma HeLa. The cytotoxicity of the compounds was determined using MTT assay and DOX as a positive control [28-31].

The cytotoxic activities of the novel synthesized compounds 8-23 were estimated and the obtained results are presented in Figure 4. In general, it can be seen that all synthesized compounds exhibited cytotoxic activities against both tested cancer cell lines. Moreover, it can be seen that both cells reacted in a dose-dependent manner toward the applied concentrations. Additionally, both tested cell lines varied in their response toward different synthesized compounds. Furthermore, based on the $\mathrm{IC}_{50}$ values (Table 1) obtained for the tested compounds, it can be seen that cytotoxic activities ranged from very strong to non-cytotoxic. Compounds 13 and 19 exhibited the most potent cytotoxic effect (very strong activity) with $\mathrm{IC}_{50} 8.78 \pm 0.7,5.16 \pm 0.4 \mu \mathrm{g} / \mathrm{mL}$, and $15.32 \pm 1.2$ and $4.26 \pm 0.3 \mu \mathrm{g} / \mathrm{mL}$ for HepG2 and HeLa cells, respectively. Furthermore, it can be noticed that Cpd. 19 exhibited more or less stronger activity similar to DOX towards HepG2 cells, (IC $505.16 \pm 0.4$ and $4.50 \pm 0.2 \mu \mathrm{g} / \mathrm{mL}$, respectively). On the other hand, it was stronger by about $23.5 \%$ than DOX against HeLa cells ( $4.50 \pm$ 
0.2 and $5.57 \pm 0.4 \mu \mathrm{g} / \mathrm{mL}$, respectively). Additionally, Cpd. 18 showed very strong activity towards HeLa cells with $\mathrm{IC}_{50}$ value of $7.67 \pm 0.6 \mu \mathrm{g} / \mathrm{mL}$, while it exhibited strong activity towards HepG2 cells $\left(\mathrm{IC}_{50} 16.70 \pm 1.3 \mu \mathrm{g} / \mathrm{mL}\right)$. Moreover, Cpd. 14 showed strong cytotoxic activities towards both tested cell lines ( $\mathrm{IC}_{50}$ values $12.20 \pm 1.0$ and $19.44 \pm 1.4 \mu \mathrm{g} / \mathrm{mL}$ for HepG2 and HeLa cells, respectively). Meanwhile, Cpds. 16 and 22 showed moderate and strong activities towards both cell lines. Cpd. 16 showed $\mathrm{IC}_{50}$ value of $33.45 \pm 2.3$ and $10.37 \pm 0.9 \mu \mathrm{g} / \mathrm{mL}$ against HepG2 and HeLa cells, respectively. Also, Cpd. 22 showed $\mathrm{IC}_{50}$ of $26.64 \pm 1.9$ and $9.33 \pm 0.8 \mu \mathrm{g} / \mathrm{mL}$ for HepG2 and HeLa cells, respectively. On the other hand, Cpd. 17 showed strong activity towards HepG2 cells $\left(\mathrm{IC}_{50} 20.00 \pm 1.7 \mu \mathrm{g} / \mathrm{mL}\right)$ and moderate activity towards HeLa cells $\left(\mathrm{IC}_{50} 35.58 \pm 2.6 \mu \mathrm{g} / \mathrm{mL}\right)$. Finally, Cpds. 9, 10, 11, 12, 15a, b, 17, 20, 21 and 23 showed activities ranging from moderate to non-cytotoxic, with $\mathrm{IC}_{50}$ values ranging from $24.83 \pm 1.8$ to $>100 \mu \mathrm{g} / \mathrm{mL}$.

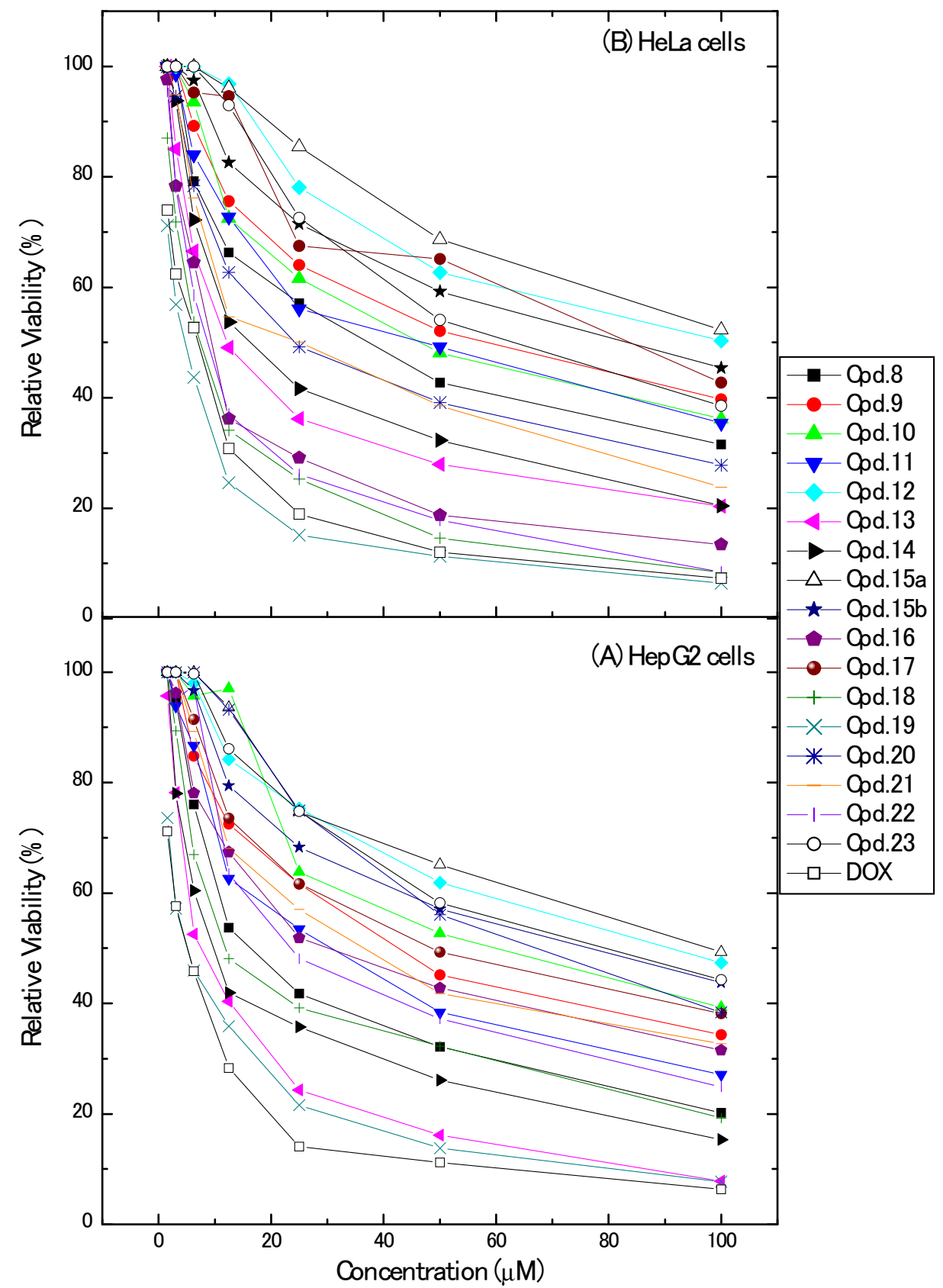

Figure 4. Relative viabilities of HepG2 and HeLa cells as affected by different synthesized compounds. 
Table 1. $\mathrm{IC}_{50}$ values obtained for the tested compounds against both HepG2 and HeLa cell lines.

\begin{tabular}{|c|c|c|}
\hline & & \\
\hline \multirow{2}{*}{ Compound } & \multicolumn{2}{|c|}{$\mathrm{IC}_{50}(\mu \mathrm{M}) *$} \\
\hline & HepG2 & HeLa \\
\hline 8 & $20.00 \pm 1.7$ & $35.58 \pm 2.6$ \\
\hline 9 & $42.95 \pm 3.2$ & $55.00 \pm 3.7$ \\
\hline 10 & $56.57 \pm 3.4$ & $47.02 \pm 3.4$ \\
\hline 11 & $30.22 \pm 2.1$ & $43.64 \pm 3.3$ \\
\hline 12 & $83.82 \pm 4.5$ & $89.72 \pm 4.7$ \\
\hline 13 & $8.87 \pm 0.70$ & $15.32 \pm 1.2$ \\
\hline 14 & $12.20 \pm 1.0$ & $19.44 \pm 1.4$ \\
\hline $15 a$ & $90.05 \pm 5.1$ & $>100$ \\
\hline $15 b$ & $68.19 \pm 3.7$ & $75.05 \pm 4.5$ \\
\hline 16 & $33.45 \pm 2.3$ & $10.37 \pm 0.9$ \\
\hline 17 & $49.66 \pm 3.2$ & $65.91 \pm 4.1$ \\
\hline 18 & $16.70 \pm 1.3$ & $7.67 \pm 0.60$ \\
\hline 19 & $5.16 \pm 0.40$ & $4.26 \pm 0.30$ \\
\hline 20 & $64.39 \pm 3.6$ & $28.15 \pm 2.2$ \\
\hline 21 & $37.42 \pm 2.5$ & $24.83 \pm 1.8$ \\
\hline 22 & $26.64 \pm 1.9$ & $9.33 \pm 0.80$ \\
\hline 23 & $73.48 \pm 4.0$ & $62.07 \pm 3.9$ \\
\hline Doxorubicin & $4.50 \pm 0.20$ & $5.57 \pm 0.40$ \\
\hline
\end{tabular}

\section{Discussion}

During current work, multi-component reaction strategy was used to synthesize of compound 8, which was used as a building block for preparing $\mathbf{1 6}$ new derivatives. The cytotoxic potential of the new prepared compounds has been evaluated against HepG2 and HeLa cells. Results obtained showed potential cytotoxic activities against both cell lines. Compounds $\mathbf{1 3}$ and $\mathbf{1 9}$ showed the most cytotoxic effects ( $\mathrm{IC}_{50} 8.78 \pm 0.7$ and $5.16 \pm 0.4 \mu \mathrm{g} / \mathrm{mL}$, for HepG2 cells, and $15.32 \pm 1.2$ and $4.26 \pm 0.3 \mu \mathrm{g} / \mathrm{mL}$ for HeLa cells, respectively). Also, results showed that both tested cell lines varied in their response toward different synthesized compounds. This can be attributed to the inherent differences in both cell lines in terms of membrane structure and organization, hence different cell lines react differently towards different compounds [32-35].

Different activities of the prepared compounds may be attributed to the structure-activity relationship of these compounds. It can be seen that conversion of Cpd. 12 to 13,14 and 16, 18, 19 and 22 altered the cytotoxicity from weak to moderate and strong activity towards two cell lines. This explained due to the introduction of two more nitrile groups, which significantly increased the activity. Compound $\mathbf{1 4}$ exhibited very strong activity due to the entity of the $\mathrm{SH}$ and $\mathrm{NH}$ groups, which may be added to any unsaturated group in DNA (thia or aza Michael addition) or the formation of hydrogen bonds with either one of the nucleo-bases of the DNA, thus causing DNA damage. Furthermore, the cytotoxicity of $\mathbf{C p d} \mathbf{1 6}$ may be due to the intermolecular hydrogen bonding of $\mathrm{NH}$ and $\mathrm{NH}_{2}$ groups with DNA moieties. Additionally, conversion of $\mathrm{Cpd}$. 16 to 18, 19 and 22 increased their cytotoxic activities against both cell lines. Introducing thiophene ring increases the cytotoxic effect of Cpd. 18 beside the effect of the pyrazole ring and the trifluoromethyl group. Additionally, introducing pyrazole ring bearing $\mathrm{NH}_{2}$ group to $\mathrm{Cpd} .16$ increases the cytotoxic effect of $\mathbf{C p d} .19$ to very strong effect against both cell lines. The introduction of chloroiso- indoline-1,3-dione increases the cytotoxic effects of Cpd. 22. The chloro- group, with more electron withdrawing properties, may be the crucial for tumor cell inhibition beside the effect of the isoindoline-1,3-dioneas moderate cytokine inhibitor in cancer cells. 


\section{Materials and Methods}

\subsection{Chemistry}

"Melting points reported are inaccurate. IR spectra were registered on Shimadzu FT-IR 8300 E (Shimadzu Corporation, Kyoto, Japan) spectrophotometer using the (KBr) disk technique. ${ }^{1} \mathrm{H}-\mathrm{NMR}$ spectra were determined on a Varian Spectrophotometer at $400 \mathrm{MHz}$ using (TMS) as an internal reference and DMSO- $\mathrm{d}_{6}$ as solvent using (TMS) as internal standard. All chemical shifts $(\delta)$ are uttered in ppm. The mass spectra were determined using (MP) model MS-5988 and Shimadzu single focusing mass spectrophotometer $(70 \mathrm{eV})$. Elemental analysis was investigated by Elemental analyzer Vario EL III".

4.1.1. Synthesis of 4-(3-(4-fluorophenyl)-1-phenyl-1H-pyrazol-4-yl)-2-hydroxy-6-(naphthalen-1-yl)nicotinenitrile (8)

A mixture of 1-acetyl naphthalene $(\mathbf{A})(1.7 \mathrm{~g}, 0.01 \mathrm{~mol})$, ethyl cyanoacetate $(1.3 \mathrm{~g}, 0.01 \mathrm{~mol})$, aldehyde (B) $(3.6 \mathrm{~g}, 0.01 \mathrm{~mol})$, ammonium acetate $(5.40 \mathrm{~g}, 0.07 \mathrm{~mol})$ and three drops of piperidine in ethanol $(20 \mathrm{~mL})$ was heated under reflux for $3 \mathrm{~h}$. The obtained precipitate was filtered off, washed with cold water, dried and crystallized from ethanol/dioxane to give compound 8 . Yield 75\%, yellow powder, m.p. $>300{ }^{\circ} \mathrm{C}$; IR $(\mathrm{KBr}): v\left(\mathrm{~cm}^{-1}\right) 3159(\mathrm{OH}), 2220(\mathrm{C} \equiv \mathrm{N}), 1647(\mathrm{C}=\mathrm{N}) ;{ }^{1} \mathrm{H}-\mathrm{NMR}\left(\mathrm{DMSO}-\mathrm{d}_{6}\right): \delta$ (ppm) 12.89 (s, 1H, OH, disappeared by $\left.\mathrm{D}_{2} \mathrm{O}\right), 9.80$ (s, 1H, pyrazole-H), 8.39-7.78 (m, 7H, Ar- $\mathrm{H}$ for naphthalene), $7.75-7.37(\mathrm{~m}, 10 \mathrm{H}, \mathrm{Ar}-\mathrm{H}) .{ }^{13} \mathrm{C}$ NMR $\left(\mathrm{DMSO}-\mathrm{d}_{6}\right): \delta(\mathrm{ppm}) 149.8(\mathrm{C}-\mathrm{OH}), 139.3(\mathrm{C}=\mathrm{N})$, $119.3(\mathrm{C} \equiv \mathrm{N}), 139.4,134.3,133.8,133.5,131.6,131.2,131.0,130.9,130.4,130.3,130.2,129.9,129.4,129.3$, 129.2, 129.1, 128.9, 128.2, 127.8, 127.6, 127.0, 125.6, 125.1, 117.4, 114.8 (Ar-CH), 40.6, 39.9 (aliph-C); MS m/z (ESI): 482 [M+] (22), 465 (21), 440 (12), 237 (100), 204; Anal. Calcd. for $\mathrm{C}_{31} \mathrm{H}_{19} \mathrm{FN}_{4} \mathrm{O}$ (482.50): C, 77.17; H, 3.97; N, 11.61. Found C, 76.98; H, 3.78; N, 11.52\%.

4.1.2. Synthesis of ethyl 2-(3-cyano-4-(3-(4-fluorophenyl)-1-phenyl-1H-pyrazol-4-yl)-6-(naphthalene-1yl)-2-oxopyridin-1(2H)-yl)acetate (9)

A mixture of 8 ( $4.84 \mathrm{~g}, 0.01 \mathrm{~mol})$, ethylchloroacetate $(1.22 \mathrm{~g}, 0.01 \mathrm{~mol})$ and $\mathrm{K}_{2} \mathrm{CO}_{3}(2.2 \mathrm{~g}, 0.015 \mathrm{~mol})$ in $\left(\mathrm{CH}_{3}\right)_{2} \mathrm{O}(40 \mathrm{~mL})$ was heated under reflux for $24 \mathrm{~h}$, concentrated and poured on water; the obtained precipitate was collected by filteration off, dried and crystallized from EtOH/dioxane to give 9. Yield 74\%, m.p. 158-160 ${ }^{\circ} \mathrm{C}$; IR (KBr): $v\left(\mathrm{~cm}^{-1}\right) 2204(\mathrm{C} \equiv \mathrm{N}), 1751$ (C=O ester), 1651 (C=O pyridine); ${ }^{1} \mathrm{H}-\mathrm{NMR}$ $\left(\mathrm{DMSO}_{\mathrm{d}}\right): \delta(\mathrm{ppm}) 9.15(\mathrm{~s}, 1 \mathrm{H}$, pyrazole-5H), 8.10-7.49 (m, 7H, Ar-H for naphthalene), 7.48-7.33 (m, $10 \mathrm{H}, \mathrm{Ar}-\mathrm{H}), 4.16$ (q, 2H, $-\mathrm{CH}_{2}$ ester), $3.40\left(\mathrm{~s}, 2 \mathrm{H},-\mathrm{CH}_{2}\right), 1.20$ (t, 3H, $-\mathrm{CH}_{3}$, ester); MS m/z (ESI): 568 [M $\left.{ }^{+}\right]$ (2.5), 495 (65), 237 (80), 127 (100); Anal. Calcd. for $\mathrm{C}_{35} \mathrm{H}_{25} \mathrm{FN}_{4} \mathrm{O}_{3}$ (568.60): C, 73.93; H, 4.43, N, 9.85. Found C, 73.80; H, 4.21; N, 9.64\%.

4.1.3. Synthesis of 8-(3-(4-fluorophenyl)-1-phenyl-1H-pyrazol-4-yl)-6-(naphthalen-1-yl)-3-oxo-3,4dihydro-2H-pyrido[2,1-c][1,2,4] triazine-9-carbonitrile (10)

A mixture of $9(5.7 \mathrm{~g}, 0.01 \mathrm{~mol}), \mathrm{NH}_{2} \mathrm{NH}_{2} \eta \mathrm{H}_{2} \mathrm{O}(2 \mathrm{~mL}, 0.04 \mathrm{~mol})$ and $\mathrm{EtOH}(20 \mathrm{~mL})$ was heated under reflux for $3 \mathrm{~h}$. The outward appearance solid was filtered off, dried and crystallized from EtOH/dioxane to give 10. Yield 71\%, yellow powder, m.p. > $300{ }^{\circ} \mathrm{C}$; IR (KBr): $v\left(\mathrm{~cm}^{-1}\right) 3209(\mathrm{NH})$, $2218(\mathrm{C} \equiv \mathrm{N}), 1647(\mathrm{C}=\mathrm{O}) ;{ }^{1} \mathrm{H}-\mathrm{NMR}\left(\mathrm{DMSO}_{\mathrm{d}}\right): \delta(\mathrm{ppm}) 12.38\left(\mathrm{~s}, 1 \mathrm{H}, \mathrm{NH}\right.$, disappeared in $\left.\mathrm{D}_{2} \mathrm{O}\right), 9.13(\mathrm{~s}$, $1 \mathrm{H}$, pyrazole-5H), 8.87-7.65 (m, 7H, Ar-H for naphthalene), 7.63-6.85 (m, 10H, Ar-H), $6.10\left(\mathrm{~s}, 2 \mathrm{H}, \mathrm{CH}_{2}\right)$. ${ }^{13}$ C-NMR (DMSO-d $\left.)_{6}\right):(\mathrm{ppm}) 165.8(\mathrm{C}=\mathrm{O}), 139.7(\mathrm{C}=\mathrm{N}), 136.1(\mathrm{C}=\mathrm{N}), 133.8,133.4,131.7,130.9,130.8$, 130.7, 130.6, 130.3, 130.2, 130.1, 129.9, 129.7, 129.2, 129.1, 128.8, 128.5, 128.1, 127.3, 126.8, 125.8, 125.6, 119.2, 119.1, 118.9, 118.5, $117.6(\mathrm{Ar}-\mathrm{CH}), 119.3(\mathrm{C} \equiv \mathrm{N}), 40.5,39.9(2 \mathrm{CH}), 17.6\left(\mathrm{CH}_{2}\right) ; \mathrm{MS} m / z$ (ESI): 519 [M+ $\left.\mathrm{M}^{+} \mathrm{OH}\right](82), 393$ (64), 284 (100), 237 (68), 127 (56); Anal. Calcd. for $\mathrm{C}_{33} \mathrm{H}_{21} \mathrm{FN}_{6} \mathrm{O}$ (536.50): C, 73.87; H, 3.94; N, 15.66. Found C, 73.68; H, 3.24; N, 15.06\%. 
4.1.4. Synthesis of 5-(3-(4-fluorophenyl)-1-phenyl-1H-pyrazol-4-yl)-7-(naphthalen-1-yl)-2-oxo-1,2dihydro-1,8-naphthyridine-3-carbonitrile (11)

Refluxing of compound 8 ( $4.84 \mathrm{~g}, 0.01 \mathrm{~mol})$ with malononitrile $(0.015 \mathrm{~mol})$ in ethanol $(20 \mathrm{~mL})$ in the presence of drops of TEA for $5 \mathrm{~h}$, then cooled, poured on ice/water, neutralized with drops of conc. $\mathrm{HCl}$. The obtained solid was collected by filtration, crystallized from EtOH/dioxane to afford 11. Yield $71 \%$, pale brown powder, m.p. $>300{ }^{\circ} \mathrm{C}$; $\mathrm{IR}(\mathrm{KBr}): v\left(\mathrm{~cm}^{-1}\right) 3386,3273\left(\mathrm{NH}_{2}\right), 3158(\mathrm{NH})$, $2218(\mathrm{C} \equiv \mathrm{N}), 1646(\mathrm{C}=\mathrm{O}),{ }^{1} \mathrm{H}-\mathrm{NMR}\left(\mathrm{DMSO}_{6}\right): \delta(\mathrm{ppm}) 12.89\left(\mathrm{~s}, 1 \mathrm{H}, \mathrm{NH}\right.$, disappeared by $\left.\mathrm{D}_{2} \mathrm{O}\right), 9.08(\mathrm{~s}$, $1 \mathrm{H}$, pyrazole- $5 \mathrm{H}), 8.07-7.61\left(\mathrm{~m}, 7 \mathrm{H}, \mathrm{Ar}-\mathrm{H}\right.$ for naphthalene), 7.60-7.37 (m, 10H, Ar-H), $6.22\left(\mathrm{~s}, 2 \mathrm{H}, \mathrm{NH}_{2}\right.$, disappeared in $\left.\mathrm{D}_{2} \mathrm{O}\right) .{ }^{13} \mathrm{C}-\mathrm{NMR}\left(\mathrm{DMSO}_{6}\right): \delta(\mathrm{ppm}) 149.9(\mathrm{C}=\mathrm{O}), 139.3(\mathrm{C}=\mathrm{N}), 133.8,133.5,131.2$, 131.1, 131.00 (2), 130.9, 130.4, 130.3 (2), 130.2, 129.9 (2), 129.4, 129.1, 128.9 (2), 128.2, 127.8(2), 127.6, 127.1 (2), 125.6, 125.2 (2), 117.4, 116.8, 110.0 (Ar-CH), $119.3(\mathrm{C} \equiv \mathrm{N}), 40.6,39.9(2 \mathrm{CH}) ; \mathrm{MS} m / z$ (ESI): 532 [M ${ }^{+}-$ $\mathrm{NH}_{3}$ ] (82), 516 (76), 440 (28), 310 (20), 237 (100); Anal. Calcd. for $\mathrm{C}_{34} \mathrm{H}_{21} \mathrm{FN}_{6} \mathrm{O}$ (548.50): C, 74.44; $\mathrm{H}$, $3.89 ; \mathrm{N}, 15.32$. Found $\mathrm{C}, 74.24 ; \mathrm{H}, 3.25 ; \mathrm{N}, 14.98 \%$.

4.1.5. Synthesis of 2-chloro-4-(3-(4-fluorophenyl)-1-phenyl-1H-pyrazol-4-yl)-6-(naphthalen-1-yl)nicotinenitrile (12)

A mixture of $8(4.82 \mathrm{~g}, 0.01 \mathrm{~mol}), \mathrm{PCl}_{5}(3 \mathrm{~g}, 0.03 \mathrm{~mol})$ and $\mathrm{POCl}_{3}(5 \mathrm{~mL}, 0.03 \mathrm{~mol})$ was heated under reflux for $8 \mathrm{~h}$, then it was poured on crushed ice. The formed solid was filtered off, dried and crystallized from EtOH/dioxane to give 12. Yield 61\%, yellow powder, m.p. $164-166^{\circ} \mathrm{C} ; \mathrm{IR}(\mathrm{KBr}): v$ $\left(\mathrm{cm}^{-1}\right) 2227(\mathrm{C} \equiv \mathrm{N}), 1628(\mathrm{C}=\mathrm{N}) ;{ }^{1} \mathrm{H}-\mathrm{NMR}\left(\mathrm{DMSO}_{6}\right): \delta(\mathrm{ppm}) 9.16(\mathrm{~s}, 1 \mathrm{H}$, pyrazole-5H), 8.35-7.63 (m, 7H, Ar-H for naphthalene), 7.61-7.39 (m, 10H, Ar-H). ${ }^{13} \mathrm{C}-\mathrm{NMR}$ (DMSO-d 6 ): $\delta$ (ppm) 152.7, 150.0, 148.4, 139.2 (C=N), 135.3(C=N), 133.8, 131.5, 131.0, 130.4, 130.3, 130.2, 129.8, 129.5, $129.2(2), 129.1,127.9$, 127.6, 126.9, $125.8(2), 125.4,125.1,119.3(\mathrm{C} \equiv \mathrm{N}), 116.6,115.5,107.8(\mathrm{Ar}-\mathrm{CH}), 40.6,39.9(2 \mathrm{CH}) ; \mathrm{MS} \mathrm{m} / \mathrm{z}$ (ESI): 503 [M $\mathrm{M}^{+}$2] (6), 501 [M+1 (50), 465 (100), 237 (82); Anal. Calcd. for $\mathrm{C}_{31} \mathrm{H}_{18} \mathrm{ClFN}_{4}$ (500.90): $\mathrm{C}$, 74.32; H, 3.62; N, 11.84. Found C, 74.12; H, 3.26; N, $11.42 \%$.

4.1.6. Synthesis of 2-[4-(3-(4-fluorophenyl)-1-phenyl-1H-pyrazol-4-yl)-6-(naphthalen-1-yl)-3-cyanopyridinyl]malononitrile (13)

To a solution of $12(5.0 \mathrm{~g}, 0.01 \mathrm{~mol})$ in EtOH $(20 \mathrm{~mL})$, malononitrile $(0.01 \mathrm{~mol})$ and TEA $(1 \mathrm{~mL})$ were added. The reaction mixture was heated under for $3 \mathrm{~h}$. After cooling, it was poured on water and neutralized with diluted $\mathrm{HCl}$. The obtained solid was separated by filtration, washed with water, dried and crystallized from EtOH/dioxane to yield 13. Yield 76\%, pale brown powder, m.p. 194-196 ${ }^{\circ} \mathrm{C}$; IR $(\mathrm{KBr}): v\left(\mathrm{~cm}^{-1}\right) 2203(\mathrm{C} \equiv \mathrm{N}),{ }^{1} \mathrm{H}-\mathrm{NMR}\left(\mathrm{DMSO}_{-} \mathrm{d}_{6}\right): \delta(\mathrm{ppm}) 9.15(\mathrm{~s}, 1 \mathrm{H}$, pyrazole-5H), 8.11-7.66 (m, 7H, Ar-H for naphthalene), 7.65-7.36 (m, 10H, Ar-H), 7.07 (s, 1H, CH of CH(CN) $\left.)_{2}\right), \mathrm{MS} \mathrm{m} / z$ (ESI): 530 [M+ $\mathrm{M}^{+}$(12), 440 (100), 237 (76), 204 (31); Anal. Calcd. for $\mathrm{C}_{34} \mathrm{H}_{19} \mathrm{FN}_{6}$ (530.50): C, 76.97; H, 3.61; N, 15.84. Found C, $76.78 ; \mathrm{H}, 3.42 ; \mathrm{N}, 15.24 \%$.

\subsubsection{Synthesis of $\mathbf{1 4}$ and $\mathbf{1 5 a} \mathbf{a} \mathbf{b}$}

A mixture of 2-chloronicotinonitrile $12(5.0 \mathrm{~g}, 0.01 \mathrm{~mol})$ and the appropriate amine, namely, o-aminothiophenol, morpholine or 2-methylpiperidine $(0.01 \mathrm{~mol})$ in EtOH $(20 \mathrm{~mL})$ was heated under reflux for $3 \mathrm{~h}$, then it was poured on cold water, filtered off and crystallized from $\mathrm{EtOH} /$ dioxane to afford 14 and $15 a, b$, respectively.

4-(3-(4-Fluorophenyl)-1-phenyl-1H-pyrazol-4-yl)-2-(2-mercaptophenylamino)-6-(naphthalen-1-yl) nicotinonitrile (14). Yield 74\%, brown powder, m.p. 108-110 ${ }^{\circ} \mathrm{C}$; IR $(\mathrm{KBr}): v\left(\mathrm{~cm}^{-1}\right) 3330(\mathrm{NH})$, $2208(\mathrm{C} \equiv \mathrm{N}),{ }^{1} \mathrm{H}-\mathrm{NMR}\left(\mathrm{DMSO}_{\mathrm{d}}\right)$ ) $\delta(\mathrm{ppm}) 9.29(\mathrm{~s}, 1 \mathrm{H}$, pyrazole-5H), 9.06-8.54 $(\mathrm{m}, 4 \mathrm{H}, \mathrm{Ar}-\mathrm{H}$, thionyl-H), 8.26-7.66 (m, 7H, Ar-H for naphthalene), 7.60-6.66 (m, 10H, Ar-H), $3.34(\mathrm{~s}, 1 \mathrm{H}, \mathrm{NH}$,

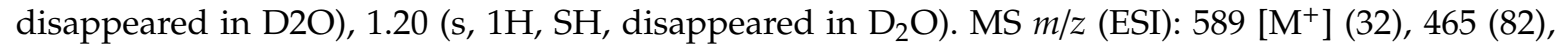
441 (62), 237 (100), 127(12), 124 (20); Anal. Calcd. for $\mathrm{C}_{37} \mathrm{H}_{24} \mathrm{FN}_{5} \mathrm{O}$ (589.60): C, 75.36, H, 4.10; N, 11.88. Found C, 75.18; H, 4.05; N, 11.73\%. 
4-(3-(4-Fluorophenyl)-1-phenyl-1H-pyrazol-4-yl)-2-morpholino-6-(naphthalen-1-yl)nicotinonitrile (15a). Yield 65\%, pale brown powder, m.p. 130-133 ${ }^{\circ} \mathrm{C}$; IR $(\mathrm{KBr}): v\left(\mathrm{~cm}^{-1}\right) 2226(\mathrm{C} \equiv \mathrm{N}),{ }^{1} \mathrm{H}-\mathrm{NMR}$ $\left(\right.$ DMSO-d $\left._{6}\right): \delta(\mathrm{ppm}) 9.16(\mathrm{~s}, 1 \mathrm{H}$, pyrazole-5H), 8.71-7.56 (m, 7H, Ar-H for naphthalene), 7.55-7.15 $(\mathrm{m}, 10 \mathrm{H}, \mathrm{Ar}-\mathrm{H}), 3.76(\mathrm{t}, 4 \mathrm{H}, J=8.8 \mathrm{~Hz}), 3.05(\mathrm{t}, 4 \mathrm{H}, J=8.8 \mathrm{~Hz}), \mathrm{MS} m / z$ (ESI): 552 [M $\left.{ }^{+}\right]$(52), 465 (28), 237 (100), 230 (7), 127 (12), 87 (22); Anal. Calcd. for $\mathrm{C}_{35} \mathrm{H}_{26} \mathrm{FN}_{5} \mathrm{O}$ (551.60): C, 76.21; H, 4.75; N, 12.70. Found $\mathrm{C}, 75.98 ; \mathrm{H}, 4.26 ; \mathrm{N}, 12.31 \%$.

4-(3-(4-Fluorophenyl)-1-phenyl-1H-pyrazol-4-yl)-2-(4-methylpiperazin-1-yl)-6-(naphthalen-1-yl) nicotinonitrile (15b). Yield 61\%, brown powder, m.p. 156-158 ${ }^{\circ} \mathrm{C}$; IR $(\mathrm{KBr}): v\left(\mathrm{~cm}^{-1}\right) 2918$ (aliph-H), $2227(\mathrm{C} \equiv \mathrm{N}),{ }^{1} \mathrm{H}-\mathrm{NMR}\left(\mathrm{DMSO}_{-} \mathrm{d}_{6}\right): \delta(\mathrm{ppm}) 9.18(\mathrm{~s}, 1 \mathrm{H}$, pyrazole-5H), 8.71-7.65 $(\mathrm{m}, 7 \mathrm{H}, \mathrm{Ar}-\mathrm{H}$ for naphthalene), 7.64-7.12 (m, 10H, Ar-H), 3.30-3.25 (m, 4H, 2CH $), 2.43-2.23\left(\mathrm{~m}, 4 \mathrm{H}, 2 \mathrm{CH}_{2}\right), 2.24(\mathrm{~s}, 3 \mathrm{H}$, $\mathrm{CH}_{3}$ ), MS m/z (ESI): 564 [M $\mathrm{M}^{+}$(27), 538 (25), 439 (12), 237 (100), 100 (23); Anal. Calcd. for $\mathrm{C}_{35} \mathrm{H}_{29} \mathrm{FN}_{6}$ (564.60): C, 76.58, H, 5.18; N, 14.88. Found C, 75.98; H, 4.92; N, 14.72\%.

4.1.8. Synthesis of 4-(3-(4-Fluorophenyl)-1-phenyl-1H-pyrazol-4-yl)-2-hydrazinyl-6-(naphthalen-1-yl) nicotinonitrile (16)

A mixture of the 2-chloronicotinonitrile $12(5.0 \mathrm{~g}, 0.01 \mathrm{~mol})$ and $\mathrm{NH}_{2} \mathrm{NH}_{2} \cdot \mathrm{H}_{2} \mathrm{O}(0.04 \mathrm{~mol})$ in $\mathrm{EtOH}(20 \mathrm{~mL})$ was heated under reflux for $4 \mathrm{~h}$. The obtained solid was collected by filtration, dried and crystallized from EtOH/dioxane to yield 16. Yield $86 \%$, yellow powder, m.p. $164-168{ }^{\circ} \mathrm{C}$; IR (KBr): $v\left(\mathrm{~cm}^{-1}\right)$ 3417, $3310\left(\mathrm{NH}_{2}\right), 3199(\mathrm{NH}), 2206(\mathrm{C} \equiv \mathrm{N}),{ }^{1} \mathrm{H}-\mathrm{NMR}\left(\mathrm{DMSO}_{6}\right): \delta(\mathrm{ppm}) 9.16(\mathrm{~s}$, $1 \mathrm{H}$, pyrazole- $5 \mathrm{H}), 8.35-7.97(\mathrm{~m}, 7 \mathrm{H}, \mathrm{Ar}-\mathrm{H}$ for naphthalene), 7.96-6.88 (m, 10H, Ar- $\mathrm{H}), 4.82(\mathrm{~s}, 1 \mathrm{H}$, $\mathrm{NH}$, disappeared in $\left.\mathrm{D}_{2} \mathrm{O}\right), 3.43\left(\mathrm{~s}, 2 \mathrm{H}, \mathrm{NH}_{2}\right.$, disappeared in $\left.\mathrm{D}_{2} \mathrm{O}\right) .{ }^{13} \mathrm{C}-\mathrm{NMR}\left(\mathrm{DMSO}-\mathrm{d}_{6}\right): \delta(\mathrm{ppm})$ $149.3\left(\mathrm{C}-\mathrm{NHNH}_{2}\right), 148.3,139.7(\mathrm{C} \equiv \mathrm{N}), 139.2,138.5,136.1(\mathrm{C}=\mathrm{N}), 135.3,134.0,133.8,131.7,131.5,131.0$, 130.9, 130.4, 130.2, 130.1, 129.5, 129.1, 128.1, 127.9, 127.6, 127.3, 126.9, 126.7, 126.4, 125.8, 125.4, 119.3 $(\mathrm{C} \equiv \mathrm{N}), 118.2(\mathrm{Ar}-\mathrm{CH}), 40.6,40.0(2 \mathrm{CH}) ; \mathrm{MS} m / z(\mathrm{ESI}): 496\left[\mathrm{M}^{+}\right]$(12), 465 (81), 440 (100), 237 (20), 204 (76); Anal. Calcd. for $\mathrm{C}_{31} \mathrm{H}_{21} \mathrm{FN}_{6}$ (496.55): C, 74.99; H, 4.26; N, 16.93. Found C, 74.86; H, 4.12; N, $16.78 \%$.

\subsubsection{Synthesis of $\mathbf{1 7}$ and $\mathbf{1 8}$}

A mixture of 16 ( $4.9 \mathrm{~g}, 0.01 \mathrm{~mol}$ ), acetylacetone or 4,4,4-trifluoro-1-(thiophen-2-yl)butane-1,3-dione $(0.01 \mathrm{~mol})$ in $\mathrm{EtOH}(10 \mathrm{~mL})$ and $\mathrm{AcOH}(4 \mathrm{~mL})$ was heated reflux for $3 \mathrm{~h}$. After cooling, the solid obtained was filtered off, dried and crystallized from EtOH/dioxane to afford 17 and 18, respectively.

2-(3,5-Dimethyl-1H-pyrazol-1-yl)-4-(3-(4-fluorophenyl)-1-phenyl-1H-pyrazol-4-yl)-6(naphthalen-1-yl)nicotinonitrile (17). Yield 85\%, pale orange powder, m.p. $270-272{ }^{\circ} \mathrm{C}$; IR (KBr): $v$ $\left(\mathrm{cm}^{-1}\right) 2209(\mathrm{C} \equiv \mathrm{N}), 1620(\mathrm{C}=\mathrm{N}),{ }^{1} \mathrm{H}-\mathrm{NMR}\left(\mathrm{DMSO}_{6} \mathrm{~d}_{6}\right): \delta(\mathrm{ppm}) 9.24(\mathrm{~s}, 1 \mathrm{H}$, pyrazole-5H), 8.17-7.96 (m, $7 \mathrm{H}, \mathrm{Ar}-\mathrm{H}$ for naphthalene), 7.66-7.35 (m, 10H, Ar-H), $7.25(\mathrm{~s}, 1 \mathrm{H}$, pyrazole- $4 \mathrm{H}), 2.48\left(\mathrm{~s}, 6 \mathrm{H}, 2 \mathrm{CH}_{3}\right)$; MS m/z (ESI): 560 [M $\mathrm{M}^{+}$(13), 533 (26), 438 (62), 237 (15), 95 (100); Anal. Calcd. for $\mathrm{C}_{36} \mathrm{H}_{25} \mathrm{FN}_{6}$ (560.60): C, 77.13; H, 4.49; N, 14.99. Found C, 76.92; H, 4.32; N, 14.81\%.

4-(3-(4-Fluorophenyl)-1-phenyl-1H-pyrazol-4-yl)-6-(naphthalen-1-yl)-2-(5-(thiophen-2-yl)-3-(trifluoromethyl)- $1 H$-pyrazol-1-yl)nicotinonitrile (18). Yield $82 \%$, dark yellow powder, m.p. $117-119^{\circ} \mathrm{C}$; IR $(\mathrm{KBr}): v\left(\mathrm{~cm}^{-1}\right) 2209(\mathrm{C} \equiv \mathrm{N}),{ }^{1} \mathrm{H}-\mathrm{NMR}\left(\mathrm{DMSO}_{6} \mathrm{~d}_{6}\right): \delta(\mathrm{ppm}) 8.92(\mathrm{~s}, 1 \mathrm{H}$, pyrazole-5H), 8.03-7.89 (m, 7H, Ar-H for naphthalene), 7.59-7.54 (m, 3H, thionyl-H), 7.53-7.33 (m, 10H, Ar-H), $6.88(\mathrm{~s}, 1 \mathrm{H}$, pyrazole-4H); MS m/z (ESI): 583 [M $\left.\mathrm{M}^{+}\right]$(10), 465 (72), 237 (100), 299 (8), 217 (5); Anal. Calcd. for $\mathrm{C}_{39} \mathrm{H}_{22} \mathrm{~F}_{4} \mathrm{~N}_{6} \mathrm{~S}$ (682.60): $\mathrm{C}, 68.61 ; \mathrm{H}, 3.25 ; \mathrm{N}, 12.31$. Found $\mathrm{C}, 68.02 ; \mathrm{H}, 3.12 ; \mathrm{N}, 12.03 \%$.

\subsubsection{Synthesis of $\mathbf{1 9}$ and $\mathbf{2 0}$}

A solution of $16(4.9 \mathrm{~g}, 0.01 \mathrm{~mol})$ in a mixture of $\mathrm{AcOH} / \mathrm{Ac}_{2} \mathrm{O}(10 \mathrm{~mL})$ or in glacial $\mathrm{AcOH}(10$ $\mathrm{mL}$ ) was refluxed for $2 \mathrm{~h}$, poured on ice/water, filtered off and crystallized from $\mathrm{EtOH} /$ dioxane to give 19 and 20, respectively. Also, refluxing of $19(0.5 \mathrm{~g}, 0.01 \mathrm{~mol})$ in acetic anhydride $(7 \mathrm{~mL})$ afforded compound 20.

4-(3-(4-Flurophenyl)-1-phenyl-1H-pyrazol-4-yl)-6-(naphthalen-1-yl)-1H-pyrazolo[3,4-b]pyridin-3amine (19). Yield $84 \%$, pale yellow powder, m.p. $140-143{ }^{\circ} \mathrm{C}$; IR $(\mathrm{KBr}): \vee\left(\mathrm{cm}^{-1}\right) 3425-3354\left(\mathrm{NH}_{2}\right)$, 
$3198(\mathrm{NH}),{ }^{1} \mathrm{H}-\mathrm{NMR}\left(\mathrm{DMSO}_{6} \mathrm{~d}_{6}\right): \delta(\mathrm{ppm}) 8.92(\mathrm{~s}, 1 \mathrm{H}$, pyrazole-5H), 8.22-7.90 (m, 7H, Ar-H for naphthalene), 7.66-7.34 (m, 10H, Ar-H), $5.02\left(\mathrm{~s}, 2 \mathrm{H}, \mathrm{NH} 2\right.$, disappeared in $\left.\mathrm{D}_{2} \mathrm{O}\right), 4.63(\mathrm{~s}, 1 \mathrm{H}, \mathrm{NH}$, disappeared in $\left.\mathrm{D}_{2} \mathrm{O}\right)$; $\mathrm{MS} \mathrm{m} / \mathrm{z}$ (ESI): 496 [M+1 $]$ (28), 479 (76), 244 (50), 237 (100); Anal. Calcd. for $\mathrm{C}_{31} \mathrm{H}_{21} \mathrm{FN}_{6}$ (496.52): C, 74.99; H, 4.26; N, 16.93. Found C, 74.76; H, 4.15; N, 16.82\%.

$\mathrm{N}$-(4-(3-(4-Flurophenyl)-1-phenyl-1H-pyrazol-4-yl)-6-(naphthalen-1-yl)-1H-pyrazolo-[3,4-b] pyridin-3-yl)acetamide (20). Yield 78\%, yellow powder, m.p. $138-140{ }^{\circ} \mathrm{C} ; \mathrm{IR}(\mathrm{KBr}): \vee\left(\mathrm{cm}^{-1}\right) 3196$ $(\mathrm{NH}), 1690(\mathrm{C}=\mathrm{O}),{ }^{1} \mathrm{H}-\mathrm{NMR}\left(\mathrm{DMSO}_{6} \mathrm{~d}_{6}\right): \delta(\mathrm{ppm}) 12.37 \& 10.31(\mathrm{~s}, \mathrm{NH}, \mathrm{OH}), 8.88(\mathrm{~s}, 1 \mathrm{H}$, pyrazole- $5 \mathrm{H})$, 7.98-7.59 (m, 7H, Ar-H for naphthalene), 7.57-6.88 (m, 10H, Ar-H), 4.82 (s, 1H, NH, disappeared in $\left.\mathrm{D}_{2} \mathrm{O}\right), 2.73$ (s, 3H, acetyl); MS m/z (ESI): 538 [M $\mathrm{M}^{+}$(20), 479 (36), 244 (20), 237 (100); Anal. Calcd. for $\mathrm{C}_{33} \mathrm{H}_{23} \mathrm{FN}_{6} \mathrm{O}$ (538.59): C, 73.59; H, 4.30; N, 15.60. Found C, 73.28; H, 4.19; N, 15.32\%.

4.1.11. Synthesis of N-(4-chlorobenzylidene)-4-(3-(4-fluorophenyl)-1-phenyl-1H-pyrazol-4-yl)-6(naphthalen-1-yl)-1H-pyrazolo[3,4-b]pyridine-3-amine (21)

A solution of 16 or $19(0.01 \mathrm{~mol})$ in $\mathrm{AcOH}(10 \mathrm{~mL})$ in the presence of 4-chlorobenzaldehyde $(0.01$ $\mathrm{mol}$ ) was heated under reflux for $2 \mathrm{~h}$, left to precipitate, filtered and crystallized from EtOH/ dioxane to afford 21. Yield 58\%, yellow powder, m.p. 158-160 ${ }^{\circ} \mathrm{C}$; IR $(\mathrm{KBr}): v\left(\mathrm{~cm}^{-1}\right) 3192(\mathrm{NH}),{ }^{1} \mathrm{H}-\mathrm{NMR}$ $\left(\right.$ DMSO-d $\left._{6}\right): \delta(\mathrm{ppm}) 9.89(\mathrm{~s}, 1 \mathrm{H}$, pyrazole-5H), $9.06(\mathrm{~s}, 1 \mathrm{H}, \mathrm{N}=\mathrm{C}-\mathrm{H}), 8.87-7.56(\mathrm{~m}, 7 \mathrm{H}, \mathrm{Ar}-\mathrm{H}$ for naphthalene), 7.52-6.88 (m, 14H, Ar-H), $4.82\left(\mathrm{~s}, 1 \mathrm{H}, \mathrm{NH}\right.$, disappeared in $\left.\mathrm{D}_{2} \mathrm{O}\right) ; \mathrm{MS} m / z(\mathrm{ESI}): 621\left[\mathrm{M}^{+}\right.$] (15), 619 (48), 479 (20), 237 (80), 139 (35), 137 (100); Anal. Calcd. for $\mathrm{C}_{38} \mathrm{H}_{24} \mathrm{ClFN}_{6}$ (619.10): C, 73.72; $\mathrm{H}$, 3.91; N, 13.57. Found C, 73.25; H, 3.82; N, 13.27\%.

4.1.12. Synthesis of 2-(4-(3-(4-fluorophenyl)-1-phenyl-1H-pyrazol-4-yl)-6-(naphthalen-1-yl)- $1 H$ pyrazolo[3,4-b]-pyridin-3-yl)isoindoline-1,3-dione (22)

A mixture of 16 or $19(0.01 \mathrm{~mol})$ and tetrachlorophthalic anhydride $(0.01 \mathrm{~mol})$ in glacial acetic acid $(10 \mathrm{~mL})$ was refluxed for $1 \mathrm{~h}$, poured on ice water, filtered off and crystallized from EtOH/dioxane to yield 22. Yield 94\%, yellow powder, m.p. $115-117^{\circ} \mathrm{C}$; IR $(\mathrm{KBr}): v\left(\mathrm{~cm}^{-1}\right) 3196(\mathrm{NH}), 1785,1731(\mathrm{C}=\mathrm{O})$; ${ }^{1} \mathrm{H}-\mathrm{NMR}\left(\mathrm{DMSO}_{\mathrm{d}}\right): \delta(\mathrm{ppm}) 8.87(\mathrm{~s}, 1 \mathrm{H}$, pyrazole-5H), 8.04-7.56 $(\mathrm{m}, 7 \mathrm{H}$, Ar-H for naphthalene), 7.55-7.33 (m, 10H, Ar-H), $4.28\left(\mathrm{~s}, 1 \mathrm{H}, \mathrm{NH}\right.$, disappeared in $\left.\mathrm{D}_{2} \mathrm{O}\right)$; Anal. Calcd. for $\mathrm{C}_{39} \mathrm{H}_{19} \mathrm{Cl}_{4} \mathrm{FN}_{6} \mathrm{O}_{2}$ (764.42): C, 61.28; H, 2.51; N, 10.99. Found C, 61.00; H, 2.42; N, 10.89\%.

4.1.13. Synthesis of 7-(3-(4-fluorophenyl)-1-phenyl-1H-pyrazol-4-yl)-5-(naphthalen-1-yl)-3-thioxo2,3-dihydro[1,2,4]triazolo[4,3-a]pyridine-8-carbonitrile (23)

Solution of hydrazinyl derivative 16 (4.9 g, $0.01 \mathrm{~mol})$ in alcoholic $\mathrm{KOH}(10 \%, 20 \mathrm{~mL})$ and $\mathrm{CS}_{2}$ $(0.01 \mathrm{~mol})$ was refluxed for $2 \mathrm{~h}$, lift overnight, then poured on ice water, filtered off the solid obtained and crystallized from EtOH/dioxane to afford 23. Yield $47 \%$ yellow powder, m.p. $288-290^{\circ} \mathrm{C} ; \mathrm{IR}(\mathrm{KBr})$ : $v\left(\mathrm{~cm}^{-1}\right) 3192(\mathrm{NH}), 2218(\mathrm{C} \equiv \mathrm{N}), 1240(\mathrm{C}=\mathrm{S}) ;{ }^{1} \mathrm{H}-\mathrm{NMR}\left(\mathrm{DMSO}_{6}\right): \delta(\mathrm{ppm}) 8.73(\mathrm{~s}, 1 \mathrm{H}$, pyrazole-5H), 7.97-7.63 (m, 7H, Ar-H for naphthalene), 7.53-6.77 (m, 10H, Ar-H), $3.76(\mathrm{~s}, 1 \mathrm{H}, \mathrm{NH}$, disappeared in $\left.\mathrm{D}_{2} \mathrm{O}\right) .{ }^{13} \mathrm{C}-\mathrm{NMR}\left(\mathrm{DMSO}_{6}\right): \delta(\mathrm{ppm}) 148.1(\mathrm{C}=\mathrm{S}), 142.3,138.7(\mathrm{C}=\mathrm{N}), 133.8(2), 133.4(\mathrm{C}=\mathrm{N}), 131.7$ (2), 131.2, 130.6 (2), 130.1, 129.9 (2), 129.4, 129.2 (2), 128.9, 128.4 (2), 126.9, 126.4 (2), 126.3 (2), 125.9 (2), $119.1(\mathrm{C} \equiv \mathrm{N}), 110.0(\mathrm{Ar}-\mathrm{CH}), 40.5,39.9(2 \mathrm{CH}) ; \mathrm{MS} m / z(\mathrm{ESI}): 538\left[\mathrm{M}^{+}\right]$(45), 494 (18), 479 (10), 453 (50), 237 (100); Anal. Calcd. for $\mathrm{C}_{32} \mathrm{H}_{19} \mathrm{FN}_{6} \mathrm{~S}$ (538.60): C, 71.36; H, 3.56; N, 15.60. Found C, 71.31; H, 3.52; $\mathrm{N}, 15.58 \%$.

\subsection{Cytotoxicity Assay}

\subsubsection{Materials and Cell Lines}

Hepatocellular carcinoma (HepG2) and cervical Carcinoma (HeLa) cell lines, ATCC, VA, USA, were used throughout the work. All used chemicals and reagents were of high purity-cell culture grade. 


\subsubsection{MTT Assay}

Cytotoxic assay depends on the formation of purple formazan crystals by the action of dehydrogenase in living cells. Cells were cultured in RPMI-1640 medium supplemented with $10 \%$ fetal bovine serum, antibiotic solution ( 100 units $/ \mathrm{mL}$ penicillin, $100 \mu \mathrm{g} / \mathrm{mL}$ streptomycin) at $37^{\circ} \mathrm{C}$ in a $5 \% \mathrm{CO} 2$ incubator. Cells were seeded in a 96 -well plate $\left(10^{4}\right.$ cells/well), and the plates were incubated for $48 \mathrm{~h}$. Afterwards, cells were exposed to variable concentrations of prepared derivatives and incubation proceeded for further $24 \mathrm{~h}$. After treatment, $20 \mu \mathrm{L}$ of MTT solution $(5 \mathrm{mg} / \mathrm{mL})$ was added and incubated for $4 \mathrm{~h}$. DMSO $(100 \mu \mathrm{L} /$ well $)$ is added and the developed color density was measured at $570 \mathrm{~nm}$ using a plate reader (ELx 800, BioTek, Winuski, VT, USA). Relative cell viability was calculated as (Atreated/Auntreated) $\times 100[36,37]$. Results were compared with doxorubicin as a positive control.

\section{Conclusions}

During the current investigation, we synthesized a new building block; namely 4-(3-(4-fluorophenyl)-1-phenyl-1H-pyrazol-4-yl)-2-hydroxy-6-(naphthalen-1-yl)nicotinonitril, with the help of multicomponent reaction systems. From that compound, a series of $\mathbf{1 6}$ different nicotinonitril derivatives were synthesized, and their structural and spectral data were elucidated. Furthermore, in vitro cytotoxic activities against hepatocellular and cervical carcinoma cell lines were investigated. Obtained results revealed that different synthesized compounds showed promising in vitro cytotoxic activities against both HepG2 and HeLa cell lines. Compounds $\mathbf{1 3}$ and $\mathbf{1 9}$ showed the most potent cytotoxic effect ( $\mathrm{IC}_{50}: 8.78 \pm 0.7,5.16 \pm 0.4 \mu \mathrm{g} / \mathrm{mL}$, and $15.32 \pm 1.2$ and $4.26 \pm 0.3 \mu \mathrm{g} / \mathrm{mL}$ for HepG2 and HeLa cells, respectively.

Author Contributions: The listed authors contributed to this work as described in the following: A.A.E.-S. and A.K.E.-Z. synthesis, and interpreted the spectroscopic identification of the synthesized compounds, A.E.-G.E.A. and E.A.E. are interpreted the results, the experimental part and E.A.E. performed the revision before submission. All authors read and approved the final manuscript.

Funding: The authors are grateful to the Deanship of Scientific Research, king Saud University for funding through Vice Deanship of Scientific Research Chairs.

Acknowledgments: The authors are appreciative to Faculty of Science, Ain Shams University where the experimental part carried out in its laboratories and Faculty of Pharmaceutical, El-Masoura University to carry the anticancer activity in it.

Conflicts of Interest: The authors declare no conflict of interest.

\section{References}

1. Rajeswari, M.; Saluja, P.; Khurana, J.M. A facile and green approach for the synthesis of spiro[naphthalene-2,50-pyrimidine]-4-carbonitrile via a one-pot three-component condensation reaction using DBU as a catalyst. Rsc. Adv. 2016, 6, 1307-1312. [CrossRef]

2. El-Sayed, H.A.; Moustafa, A.H.; Haikal, A.Z.; Abu-El-Halawa, R.; El Ashry, E.H. Synthesis, antitumor and antimicrobial activities of 4-(4-chlorophenyl)-3-cyano-2-(b-o-glycosyloxy)-6-(thien-2-yl)nicotine- nitrile. Eur. J. Med. Chem. 2011, 46, 2948-2954. [CrossRef]

3. Kotb, E.R.; El-Hashash, M.A.; Salama, M.A.; Kalf, H.S.; Abdel Wahed, N.A.M. Synthesis and reactions of some novel nicotinonitrile derivatives for anticancer and antimicrobial evaluation. Acta Chim. Slov. 2009, 56, 908-919.

4. Hamdy, N.A.; Anwar, M.M.; Abu-Zied, K.M.; Awad, H.M. Synthesis, tumor inhibitory and antioxidant activity of new polyfunctionally 2-substituted 5,6,7,8-tetrahydronaphthalene derivatives containing pyridine, thioxopyridine and pyrazolopyridine moieties. Acta Polo. Pharm. Drug Res. 2013, 70, 987-1001.

5. Salem, M.S.; Sakr, S.I.; El-Senousy, W.M.; Madkour, H.M.F.; El-Senousy, W.M. Synthesis, Antibacterial, and Antiviral Evaluation of New Heterocycles Containing the Pyridine Moiety. Arch. der Pharm. 2013, 346, 766-773. [CrossRef] 
6. $\quad$ El-Sayed, N.S.; Shirazi, A.N.; El-Meligy, M.G.; El-Ziaty, A.K.; Rowley, D.; Sun, J.; Nagib, Z.A.; Parang, K. Synthesis of 4-aryl-6-indolylpyridine-3-carbonitriles and evaluation of their anti-proliferative activity. Tetra. Lett. 2014, 55, 1154-1158. [CrossRef]

7. Ruiz, J.F.M.; Kedziora, K.; Keogh, B.; Maguire, J.; Reilly, M.; Windle, H.; Kelleher, D.P.; Gilmer, J.F. A double prodrug system for colon targeting of benzenesulfonamide COX-2 inhibitors. Bioorganic Med. Chem. Lett. 2011, 21, 6636-6640. [CrossRef]

8. Balsamo, A.; Coletta, I.; Guglielmotti, A.; Landolfi, C.; Mancini, F.; Martinelli, A.; Milanese, C.; Minutolo, F.; Nencetti, S.; Orlandini, E.; et al. Synthesis of heteroaromatic analogues of (2-aryl-1-cyclopentenyl-1-alkylidene)-(arylmethyloxy)amine COX-2 inhibitors: effects on the inhibitory activity of the replacement of the cyclopentene central core with pyrazole, thiophene or isoxazole ring. Eur. J. Med. Chem. 2003, 38, 157-168. [CrossRef]

9. Karrouchi, K.; Radi, S.; Ramli, Y.; Taoufik, J.; Mabkhot, Y.N.; Al-Aizari, F.A.; Al-Aizari, F.; Ansar, M.; A Al-Aizari, F.; Ansar, M. Synthesis and Pharmacological Activities of Pyrazole Derivatives: A Review. Molecules 2018, 23, 134. [CrossRef]

10. Bekhit, A.A.; Ashour, H.M.; Ghany, Y.S.A.; Bekhit, A.E.-D.A.; Baraka, A. Synthesis and biological evaluation of some thiazolyl and thiadiazolyl derivatives of $1 \mathrm{H}$-pyrazole as anti-inflammatory antimicrobial agents. Eur. J. Med. Chem. 2008, 43, 456-463. [CrossRef]

11. Christodoulou, M.S.; Liekens, S.; Kasiotis, K.M.; Haroutounian, S.A. Novel pyrazole derivatives: Synthesis and evaluation of anti-angiogenic activity. Bioorganic Med. Chem. 2010, 18, 4338-4350. [CrossRef]

12. Bondock, S.; Fadaly, W.; Metwally, M.A. Synthesis and antimicrobial activity of some new thiazole, thiophene and pyrazole derivatives containing benzothiazole moiety. Eur. J. Med. Chem. 2010, 45, 3692-3701. [CrossRef] [PubMed]

13. Chimenti, F.; Bolasco, A.; Manna, F.; Secci, D.; Chimenti, P.; Befani, O.; Turini, P.; Giovannini, V.; Mondovi, B.; Cirilli, R.; et al. Synthesis and Selective Inhibitory Activity of 1-Acetyl-3,5-diphenyl-4,5-dihydro-(1H)-pyrazole Derivatives against Monoamine Oxidase. J. Med. Chem. 2004, 47, 2071-2074. [CrossRef]

14. Rashad, A.E.; Hegab, M.I.; Abdel-Megeid, R.E.; Micky, J.A.; Abdel-Megeid, F.M. Synthesis and antiviral evaluation of some new pyrazole and fused pyrazolopyrimidine derivatives. Bioorganic Med. Chem. 2008, 16, 7102-7106. [CrossRef]

15. Bonesi, M.; Loizzo, M.R.; Statti, G.A.; Michel, S.; Tillequin, F.; Menichini, F. The synthesis and Angiotensin Converting Enzyme (ACE) inhibitory activity of chalcones and their pyrazole derivatives. Bioorganic Med. Chem. Lett. 2010, 20, 1990-1993. [CrossRef]

16. Mahmoud, M.R.; El-Ziaty, A.K.; Abu El-Azm, F.S.M.; Ismail, M.F.; Shiba, S.A. Utility of Cyano-N-(2-oxo-1,2-dihydroindol-3-ylidene)acetohydrazide in the Synthesis of Novel Heterocycles. J. Chem. 2013, 37, 80-85. [CrossRef]

17. El-Sayed, N.S.; Shirazi, A.N.; El-Meligy, M.G.; El-Ziaty, A.K.; Nagieb, Z.A.; Parang, K.; Tiwari, R.K. Design, synthesis, and evaluation of chitosan conjugated GGRGDSK peptides as a cancer cell-targeting molecular transporter. Int. J. Boil. Macromol. 2016, 87, 611-622. [CrossRef]

18. El-Ziaty, A.K.; Shiba, S.A. Antibacterial activities of new (E) 2-cyano-3-(3,4-dimethoxyphenyl) -2-propenoylamide derivatives. Synth. Commun. 2007, 37, 4043-4057. [CrossRef]

19. Mahmoud, M.R.; Shiba, S.A.; El-Ziaty, A.K.; Abu El-Azm, F.S.M.; Ismail, M.F. Synthesis and reactions of novel 2,5-disubistituted 1,3,4-thiadiazoles. Synth. Commun. 2014, 44, 1094-1102. [CrossRef]

20. El-Shahawi, M.M.; El-Ziaty, A.K. Enaminonitrile as Building Block in Heterocyclic Synthesis: Synthesis of Novel 4H -Furo[2,3-d ][1,3]oxazin-4-one and Furo[2,3- d ]pyrimidin-4 (3H) -one Derivatives. J. Chem. 2017, 2017, 1-6. [CrossRef]

21. Mahmoud, M.R.; El-Ziaty, A.K.; Hussein, A.M. Synthesis and Spectral Characterization of Novel Thiazolopyridine and Pyrimidine Derivatives. Synth. Commun. 2013, 43, 961-978. [CrossRef]

22. Ismail, M.F.; El-Sayed, A.A. Synthesis and in-vitro antioxidant and antitumor evaluation of novel pyrazole-based heterocycles. Iran. Chem. Soc. 2019, 16, 921-937. [CrossRef]

23. Fahmy, A.F.M.; Rizk, S.A.; Hemdan, M.M.; El-Sayed, A.A.; Hassaballah, A.I. Efficient Green Synthesis and Computational Chemical Study of Some Interesting Heterocyclic Derivatives as Insecticidal Agents. J. Chem. 2018, 55, 2545-2555. [CrossRef]

24. Rizk, S.A.; El-Sayed, A.A.; Mounier, M.M.; El-Sayed, A.A. Synthesis of Novel Pyrazole Derivatives as Antineoplastic Agent. J. Chem. 2017, 54, 3358-3371. [CrossRef] 
25. Fahmy, A.F.M.; El-Sayed, A.A.; Hemdan, M.M. Multicomponent synthesis of 4-arylidene-2-phenyl5(4H)-oxazolones (azlactones) using a mechanochemical approach. Chem. Central J. 2016, 10, 59. [CrossRef]

26. Hemdan, M.M.; El-Sayed, A.A. Use of phthalimidoacetylisothiocyanate as a scaffold in synthesis of target heterocyclic systems with their antimicrobial assessment. Chem. Pharm. Bull. 2016, 64, 483-489. [CrossRef]

27. Hemdan, M.M.; El-Sayed, A.A. Synthesis of some new heterocycles derived from novel 2-(1,3-dioxisoindolin-2-yl)benzoyl isothiocyanate. J. Heterocycl. Chem. 2016, 53, 487-492. [CrossRef]

28. Metwally, M.; Gouda, M.; Harmal, A.N.; Khalil, A. Synthesis, antitumor, cytotoxic and antioxidant evaluation of some new pyrazolotriazines attached to antipyrine moiety. Eur. J. Med. Chem. 2012, 56, 254-262. [CrossRef] [PubMed]

29. Hossan, A.; Abu-Melha, H. Synthesis, mass spectroscopic studies, cytotoxicity evaluation and quantitative structure activity relationship of novel isoindolin-1,3-dione derivatives. Chem. Process Eng. Res. 2014, 21, 60-71.

30. Eissa, I.H.; El-Naggar, A.M.; El-Hashash, M.A. Design, synthesis, molecular modeling and biological evaluation of novel 1H-pyrazolo[3,4-b]pyridine derivatives as potential anticancer agents. Bioorganic Chem. 2016, 67, 43-56. [CrossRef]

31. Shaaban, S.; Negm, A.; Sobh, M.A.; Wessjohann, L.A. Organoselenocyanates and symmetrical diselenides redox modulators: Design, synthesis and biological evaluation. Eur. J. Med. Chem. 2015, 97, 190-201. [CrossRef]

32. Elsayed, E.A.; Farooq, M.; Dailin, D.; El-Enshasy, H.A.; Othman, N.Z.; Malek, R.; Danial, E.; Wadaan, M. In vitro and in vivo biological screening of kefiran polysaccharide produced by Lactobacillus kefiranofaciens. Biomed. Res. 2017, 28, 594-600.

33. Amr, A.E.-G.E.; El-Naggar, M.; Al-Omar, M.A.; Elsayed, E.A.; Abdalla, M.M. In Vitro and In Vivo Anti-Breast Cancer Activities of Some Synthesized Pyrazolinyl-estran-17-one Candidates. Molecules 2018, $23,1572$. [CrossRef]

34. Amr, A.E.-G.E.; Abo-Ghalia, M.H.; Moustafa, G.O.; Al-Omar, M.A.; Nossier, E.S.; Elsayed, E.A. Design, Synthesis and Docking Studies of Novel Macrocyclic Pentapeptides as Anticancer Multi-Targeted Kinase Inhibitors. Molecules 2018, 23, 2416. [CrossRef]

35. Dailin, D.J.; Elsayed, E.A.; Othman, N.Z.; Malek, R.; Phin, H.S.; Aziz, R.; Wadaan, M.; El Enshasy, H.A. Bioprocess development for kefiran production by Lactobacillus kefiranofaciens in semi industrial scale bioreactor. Saudi J. Biol. Sci. 2016, 23, 495-502. [CrossRef]

36. Mosmann, T. Rapid colorimetric assay for cellular growth and survival: Application to proliferation and cytotoxicity assays. J. Immunol. Methods 1983, 65, 55-63. [CrossRef]

37. Denizot, F.; Lang, R. Rapid colorimetric assay for cell growth and survival. Modifications to the tetrazolium dye procedure giving improved sensitivity and reliability. J. Immunol. Methods 1986, 89, 271-277. [CrossRef]

Sample Availability: Samples of the compounds are available from the authors.

(C) 2019 by the authors. Licensee MDPI, Basel, Switzerland. This article is an open access article distributed under the terms and conditions of the Creative Commons Attribution (CC BY) license (http://creativecommons.org/licenses/by/4.0/). 